\title{
Coexistence of a non-thermal jet and a complex ultra-fast X-ray outflow in a moderately luminous $A G N^{\star}$
}

\author{
M. Giroletti ${ }^{1}$, F. Panessa ${ }^{2}$, A. L. Longinotti ${ }^{3}$, Y. Krongold ${ }^{4}$, M. Guainazzi ${ }^{5,6}$, E. Costantini ${ }^{7}$, and M. Santos-Lleo ${ }^{5}$ \\ 1 INAF Istituto di Radioastronomia, via Gobetti 101, 40129 Bologna, Italy \\ e-mail: giroletti@ira.inaf.it \\ 2 INAF Istituto di Astrofisica e Planetologia Spaziali di Roma, via del Fosso del Cavaliere 100, 00133 Roma, Italy \\ 3 CONACYT - Instituto Nacional de Astrofísica, Óptica y Electrónica, Luis E. Erro 1, Tonantzintla, 72840 Puebla, Mexico \\ 4 Instituto de Astronomia, Universidad Nacional Autonoma de Mexico, Apartado Postal 70264, 04510 Mexico D.F., Mexico \\ 5 ESAC, PO Box 78, 28691 Villanueva de la Cañada, Madrid, Spain \\ 6 Institute of Space and Astronautical Science, 3-1-1 Yoshinodai, Chuo-ku, Sagamihara, 252-5210 Kanagawa, Japan \\ 7 SRON Netherlands Institute for Space Research, Sorbonnelaan 2, 3584 CA Utrecht, The Netherlands
}

Received 29 November 2016 / Accepted 25 January 2017

\section{ABSTRACT}

\begin{abstract}
Context. Recent XMM-Newton observations have revealed that IRAS $17020+4544$ is a very unusual example of black hole windproduced feedback by a moderately luminous AGN in a spiral galaxy.

Aims. Since the source is known to be a radio emitter, we investigated the presence and the properties of a non-thermal component. Methods. We observed IRAS 17020+4544 with the Very Long Baseline Array at 5, 8, 15, and $24 \mathrm{GHz}$ within a month of the 2014 XMM-Newton observations. We further analysed archival data taken in 2000 and 2012.

Results. We detect the source at $5 \mathrm{GHz}$ and on short baselines at $8 \mathrm{GHz}$. At 15 and $24 \mathrm{GHz}$, the source is below our baseline sensitivity for fringe fitting, indicating a lack of prominent compact features. The morphology is that of an asymmetric double, with significant diffuse emission. The spectrum between 5 and $8 \mathrm{GHz}$ is rather steep $\left(S(v) \sim v^{-(1.0 \pm 0.2)}\right)$. Our reanalysis of the archival data at 5 and $8 \mathrm{GHz}$ provides results consistent with the new observations, suggesting that flux density and structural variability are not important in this source. We put a limit on the separation speed between the main components of $<0.06 c$.

Conclusions. IRAS $17020+4544$ shows interesting features of several classes of objects: its properties are typical of compact steep spectrum sources, low power compact sources, and radio-emitting narrow line Seyfert 1 galaxies. However, it cannot be classified in any of these categories, and remains a one-of-a-kind object.
\end{abstract}

Key words. galaxies: Seyfert - galaxies: nuclei - galaxies: jets - galaxies: active

\section{Introduction}

Ionized gas outflows from the nuclei of active galaxies (active galactic nuclei, hereafter AGNs) are a relatively common phenomenon. They are of great interest since they transport mass and energy to the host galaxy in a process known as AGN feedback, which has a fundamental impact on galaxy properties and evolution (Di Matteo et al. 2005). In this context, ultrafast outflows (UFOs, see e.g. Chartas et al. 2002; Pounds et al. 2003) are particularly relevant because of their mass outflow rate $\left(0.01-1 M_{\odot} \mathrm{yr}^{-1}\right)$ and kinetic energy $\left(10^{42}-10^{45} \mathrm{erg} \mathrm{s}^{-1}\right)$. A much less common phenomenon (less than $10 \%$ of AGNs) is the presence of a pair of relativistic jets of non-thermal plasma emerging from the central nucleus, which are generally detected in the form of collimated structures at radio wavelengths. In recent years, several works have studied the coexistence of fast outflows and relativistic jets and their possible interplay (see Tombesi et al. 2014, and references therein). A connection between the two phenomena is also predicted by magnetohydrodynamic (MHD) models (e.g. Tchekhovskoy et al. 2011; Fukumura et al. 2014).

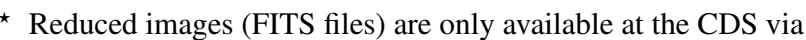
anonymous ftp to cdsarc.u-strasbg. fr (130.79.128.5) or via http://cdsarc.u-strasbg.fr/viz-bin/qcat?J/A+A/600/A87.
}

IRAS $17020+4544$ (located at RA $17 \mathrm{~h} 03 \mathrm{~m} 30.3830$ s, Dec $\left.+45^{\circ} 40^{\prime} 47^{\prime \prime} .167, z=0.0604\right)$ is an ideal target to further explore this subject. It is a bright narrow line Seyfert 1 (NLS1) spiral galaxy with black hole mass of $M_{\mathrm{BH}} \sim 5.9 \times$ $10^{6} M_{\odot}$ (Wang \& Lu 2001). We observed it in January 2014 with $X M M$-Newton, obtaining a high-resolution X-ray spectrum. Our observations reveal a series of absorption lines corresponding to at least five outflowing components with velocity in the range of $23000-33000 \mathrm{~km} \mathrm{~s}^{-1}$ (Longinotti et al. 2015). The energetics of the wind indicate that it might be able to produce feedback on the host galaxy. Evidence for such a phenomenon is also provided by the tentative presence of large-scale molecular gas outflowing at low velocity that may be associated with the fast X-ray wind (Longinotti et al., priv. comm.), as predicted by several feedback models (e.g. Faucher-Giguère \& Quataert 2012; Zubovas \& King 2012). At the same time, the presence of bright and compact radio emission indicates that this source is a radio loud (RL) NLS1 and that it also has a radio jet (Doi et al. 2007, 2011; Gu \& Chen 2010).

In the present paper, we present new high angular resolution, multifrequency Very Long Baseline Interferometry (VLBI) radio observations obtained almost simultaneously with the $X M M$-Newton observations. We further report on the analysis of archival VLBI datasets, to provide constraints on the properties of the ejection process in this source. 
The paper is organized as follows: we describe our new observations and archival data analysis in Sect. 2; we present the results in Sect. 3 and a review of the radio properties of IRAS $17020+4544$ from the literature in Sect. 4; finally, we discuss the results in Sect. 5. Throughout the paper we use a $\Lambda \mathrm{CDM}$ cosmology with $h=0.705, \Omega_{\mathrm{m}}=0.27$, and $\Omega_{\Lambda}=0.73$ (Komatsu et al. 2009). With these values, the redshift of the source corresponds to a luminosity distance of $D_{L}=269 \mathrm{Mpc}$ and to an angular scale of 1 mas $=1.16 \mathrm{pc}$; proper motion of $1 \mathrm{mas} / \mathrm{yr}$ corresponds to an apparent speed of $4.0 \mathrm{c}$. The spectral index $\alpha$ is defined such that $S(v) \sim v^{-\alpha}$ and position angles (PA) are defined positive north through east.

\section{Observations and data reduction}

\subsection{New data}

We observed IRAS $17020+4544$ with the NRAO Very Long Baseline Array (VLBA) on 2014 February 12, 20 days after the $X M M$-Newton pointing. We observed the source at 5, 8, 15, and $24 \mathrm{GHz}$, for a net integration time of $10,20,60$, and $60 \mathrm{~min}$, respectively. We recorded data with the new 2 Gbps rate offered by the VLBA, corresponding to eight baseband channels of $32 \mathrm{MHz}$ bandwidth each, in full polarization. Based on the high sensitivity provided by this observing mode and on the flux density published by Gu \& Chen (2010), we did not observe a phase calibrator for phase referencing.

We carried out the data reduction following the usual procedures in the Astronomical Image Processing System, applying calibrations for the total electronic content of the ionosphere at $v \leq 8 \mathrm{GHz}$, the Earth's orientation parameters, the correlator digital sampling, the combined system temperature and gain curves, the parallactic angle, and the instrumental single band delays. We then fringe fitted the data in order to remove residual delays, rates, and phase variations; we reanalysed an archival phase referenced dataset ( $\mathrm{Gu} \&$ Chen 2010, see also Sect. 2.2) and used the resulting image as the input model. At $5 \mathrm{GHz}$, we found solutions for most stations (all but Brewster); at $8 \mathrm{GHz}$ we found solutions only for stations forming relatively short baselines; at 15 and $24 \mathrm{GHz}$, we could not find any solution. For the 5 and $8 \mathrm{GHz}$ datasets, we then proceeded with the standard iterations of hybrid mapping, with phase only self-calibration at first, and phase and amplitude self-calibration in the final cycles.

\subsection{Archival data}

\subsubsection{June and August data}

The peak brightness and total flux density at $5 \mathrm{GHz}$ obtained in our observations are $\sim 5$ times lower than those reported at the same frequency by $\mathrm{Gu} \& \mathrm{Chen}$ (2010). Considering the nondetections at higher frequency, we are confident that the error on our measurement is not larger than the typical, conservative $10 \%$ calibration uncertainty. Therefore, in order to investigate possible amplitude and structural variability we downloaded and reanalysed the VLBA datasets presented by Gu \& Chen (2010). These datasets are BM033D and BM033E, taken on 2000 June 16 and August 21, respectively. Both datasets were obtained with two IFs of $8 \mathrm{MHz}$ bandwidth each, in dual polarization, for a total data rate of $128 \mathrm{Mbps}$. The delay, rate, and phase solutions were determined on the nearby calibrator J1713+4916 and transferred to the target source; the other steps for the calibration were identical to those described for the new observations. The total integration time on IRAS $17020+4544$ was about $100 \mathrm{~min}$ per observation.

The images at the two 2000 epochs are consistent in terms of intensity and structure, so we combined the two visibility datasets to further improve the image sensitivity and fidelity. We then produced a final image with an additional iteration of phase only and phase and amplitude self-calibration.

\subsubsection{January and February data}

We also found and downloaded two five-minute scans on IRAS $17020+4544$ obtained in the context of a large observational VLBA project at $8 \mathrm{GHz}$. These observations took place on 2012 Jan. 12 (VLBA experiment code: BC196ZQ) and 2012 Feb. 8 (code BC201AC). In both experiments, eight channels with $16 \mathrm{MHz}$ bandwidth each and single polarization were recorded and correlated. The source was bracketed by oneminute scans on $1705+456$, which we used to determine delay, rate, and phase solutions. Hancock and Saint-Croix did not produce data in either run because the calibrator was not detected (probably resolved out) on baselines to these stations. After the standard calibration, we split the data without averaging in time or frequency (other than within each of the eight baseband channels) since the phase tracking position was several hundred milliarcseconds from the position of the source determined in the other phase referencing experiment. In this way, we were able to produce images not affected by smearing and indeed we detected the source in both datasets.

\section{Results}

\subsection{New data}

We show the final images at 5 and $8 \mathrm{GHz}$ in Fig. 1. At $5 \mathrm{GHz}$, the source is extended in the east-west direction with a total flux density of about $23 \mathrm{mJy}$. A compact component with a peak brightness of $8.9 \mathrm{mJy}^{\text {beam }}{ }^{-1}$ is surrounded by diffuse emission on both sides, brighter and more extended on the western end. At $8 \mathrm{GHz}$, the overall morphology is similar. However, owing to the lack of visibility data on the longest baselines, the final sensitivity and angular resolution are poorer than at $5 \mathrm{GHz}$. The source can then be basically described as a compact asymmetric double with a brighter eastern component $\left(9.0 \mathrm{mJy}^{\text {beam }}{ }^{-1}\right.$ peak brightness) and a weaker, somewhat more diffuse western feature. There is clearly also some extended emission bridging the two features. The total flux density at $8 \mathrm{GHz}$ is $13 \mathrm{mJy}$.

We carried out a model-fit in the image plane in order to measure the brightness temperature of the main component, i.e. the eastern component labelled $E$. The brightness temperature is obtained from the formula

$T_{b}=\frac{1}{2 k_{\mathrm{B}}}\left(\frac{c}{v}\right)^{2} \frac{S(1+z)}{\pi a b}$,

where $k_{\mathrm{B}}$ is the Boltzmann constant, $c$ is the speed of light, $v$ is the observing frequency, $S$ is the total flux density of the component, $a$ and $b$ are the major and minor semi-axis of the component. In our case, $v=5.0 \mathrm{GHz}, z=0.0604$ and, for component $E, S=14 \mathrm{mJy}, a=2.5 \mathrm{mas}, b=1.0$ mas. The resulting brightness temperature is $T_{b}=1.0 \times 10^{8} \mathrm{~K}$.

We show in Fig. 2 the first quasi-simultaneous spatially resolved spectral index image of the source, based on our 5 and $8 \mathrm{GHz}$ data. Before combining the images, we convolved the $5 \mathrm{GHz}$ image with the same restoring beam of the $8 \mathrm{GHz}$ data, 

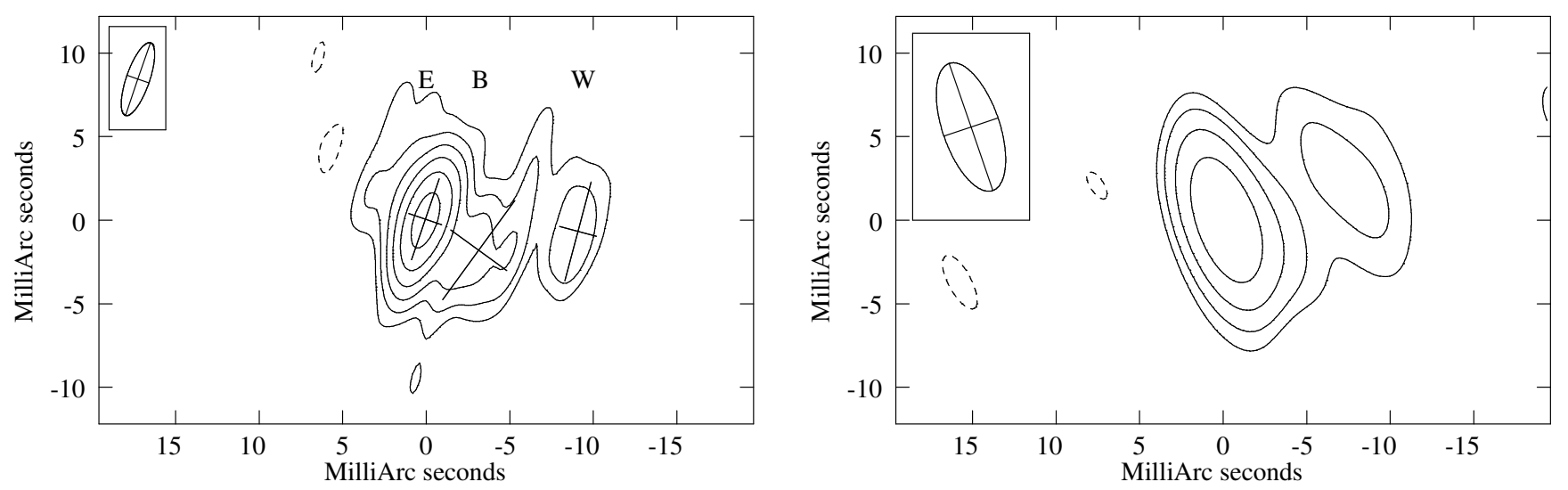

Fig. 1. VLBA images of IRAS $17020+4544$ on 2014 February 12 at $5 \mathrm{GHz}$ (left panel) and $8.4 \mathrm{GHz}$ (right panel), with contours traced at $(-1,1,2,4, \ldots, 32) \times$ the $\sim 3 \sigma$ noise level, which is 0.2 and $0.6 \mathrm{mJy}_{\text {beam }}^{-1}$ at 5 and $8 \mathrm{GHz}$, respectively. The half peak beam width is shown in the upper left corner of each panel, and is 4.6 mas $\times 1.4$ mas in position angle (PA) $-19^{\circ}$ and 8.0 mas $\times 3.4 \mathrm{mas}$ in $\mathrm{PA} 19^{\circ}$ at $5 \mathrm{and} 8 \mathrm{GHz}$, respectively. Note the unusually larger beam at $8 \mathrm{GHz}$, due to the lack of fringe detections on the longest baselines at this frequency. In the $5 \mathrm{GHz}$ image, model-fit elliptical Gaussian components are shown as crosses and labelled E, B, W, from east to west.

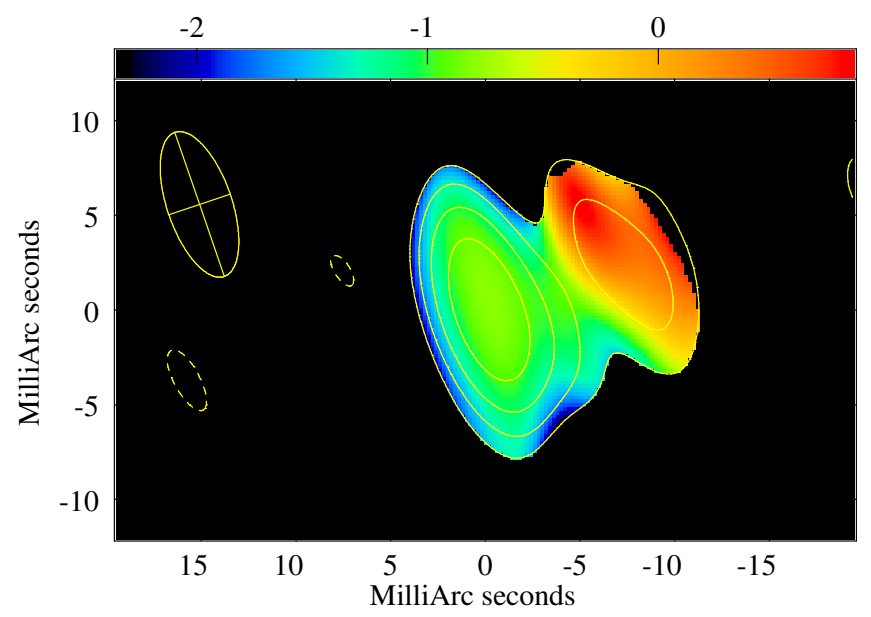

Fig. 2. Simultaneous $5-8 \mathrm{GHz}$ spectral index VLBA image of IRAS $17020+4544$ on 2014 Feb. 12. The contours show total intensity at $8 \mathrm{GHz}$; the colours show the spectral index values (note that the scale is inverted respect to what is used in the rest of the paper).

and clipped all the pixels below the $3 \sigma$ noise level in either image. As a result of this convolution, the compact eastern feature becomes "contaminated" by some additional extended emission. The image then shows a steep spectrum integrated over the eastern component and a flatter one in the western side. However, the uncertainty on the western side spectral index is larger and the eastern component is certainly affected by the presence of diffuse emission, which likely bias the results to steeper values. Indeed, if we only take the peak brightness of the component we find similar values, indicating a flat spectrum for the most compact emission region. Considering the emission detected in the entire VLBI images, the simultaneous spectral index is $\alpha=1.0 \pm 0.4$.

Finally, we estimate the upper limit to the brightness of any compact structure, based on the non-detection of the source at 24 and $15 \mathrm{GHz}$, and on the longest baselines at $8 \mathrm{GHz}$. The $5 \sigma$ VLBA sensitivity for a single baseline, channel, and polarization at 8,15 , and $24 \mathrm{GHz}$ is 12,28 , and $33 \mathrm{mJy} \mathrm{beam}^{-1}$, respectively. This assumes 10-, 5-, and 5-min integration times, respectively, for a structure with angular size of 4 mas at $8 \mathrm{GHz}$ (based on the non-detection on baselines longer than $45 M \lambda), 17$ mas at $15 \mathrm{GHz}$, and 11 mas at $24 \mathrm{GHz}$ (based on the non-detection on

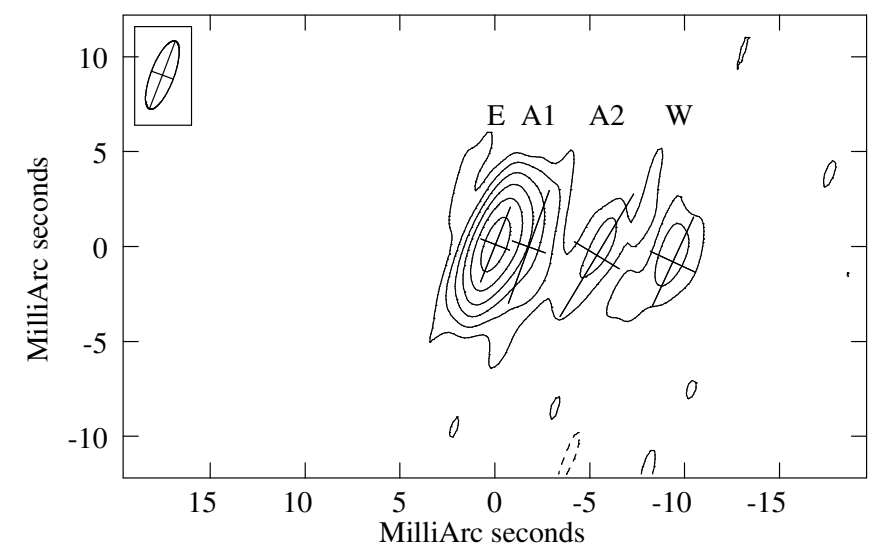

Fig. 3. VLBA image of IRAS $17020+4544$ on the combined dataset from the 2000 June and August observations at $5 \mathrm{GHz}$, with contours traced at $(-1,1,2,4, \ldots, 32) \times$ the $\sim 3 \sigma$ noise level, which is $0.27 \mathrm{mJy}$ beam $^{-1}$. The half peak beam width is shown in the upper left corner and it is 3.8 mas $\times 1.2$ mas in PA $-21^{\circ}$. Crosses and letters indicate and label model-fit elliptical Gaussian components.

any baselines in the 15 and $24 \mathrm{GHz}$ datasets). These correspond to upper limits on the brightness temperature of $2.2 \times 10^{7} \mathrm{~K}$, $1.5 \times 10^{7} \mathrm{~K}$, and $6.7 \times 10^{6} \mathrm{~K}$ at the three frequencies, respectively.

\subsection{Archival data}

We show in Fig. 3 the image obtained from the reanalysis of the combined dataset obtained from the 2000 observations. The overall morphology is consistent with both our new observations and the images reported by $\mathrm{Gu} \& \mathrm{Chen}$ (2010): the brightest component is located at the eastern end, surrounded by diffuse emission more extended in the western direction. Local peaks are embedded in this emission, with a final component located about 9 mas west of the image peak. The total flux density and the peak brightness recovered $\left(S_{5} \sim 25 \mathrm{mJy}\right.$ and $P_{5} \sim 13 \mathrm{mJy} \mathrm{beam}^{-1}$, respectively) are in much closer agreement with the 2014 data than with the values reported by Gu \& Chen (2010).

We tried to investigate on the origin of this discrepancy, but could not come up with a final explanation. We determined a flux density of $\sim 80 \mathrm{mJy}$ for the phase calibrator, which is lower than the 2.3 and $8 \mathrm{GHz}$ values reported in the VLBA calibrator 


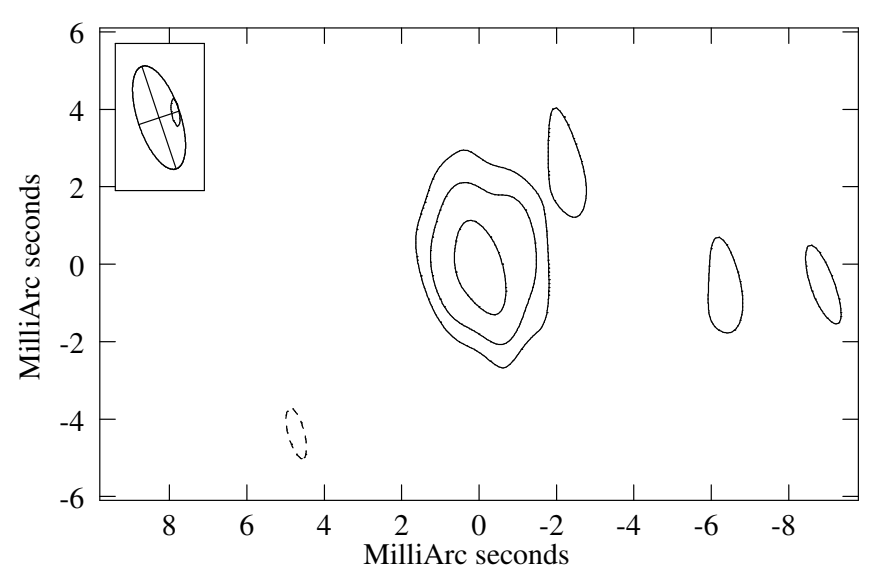

Fig. 4. VLBA $8 \mathrm{GHz}$ image of IRAS $17020+4544$ on 2012 Feb. 8, with contours traced at $(-1,1,2,4) \times$ the $\sim 3 \sigma$ noise level, which is $1.0 \mathrm{mJy}$ beam $^{-1}$. The half peak beam width is shown in the upper left corner and it is 2.8 mas $\times 1.1$ mas in PA $18^{\circ}$.

list (148 mJy on short spacings and $117 \mathrm{mJy}$ on long spacings at $2.3 \mathrm{GHz}, 217$ and $182 \mathrm{mJy}$ at $8 \mathrm{GHz}$ ); this might have prompted $\mathrm{Gu} \&$ Chen (2010) to rescale the amplitudes up by a factor of a few, resulting in the high flux density for the target. However, the flux density of 3C345 observed in the same experiment did not reveal any sign of being underestimated, and later $5 \mathrm{GHz}$ observations of J1713+4916 found flux density values closer to our measurement; also considering a fair amount of variability typical for compact sources, we do not see any reason to modify the amplitude scale for the 2000 observations of IRAS $17020+4544$.

In the $20128 \mathrm{GHz}$ experiments, we detect a slightly resolved component with an extension to the north-western side and faint diffuse emission to the west (see Fig. 4). The peak brightness is 7.4 and $5.9 \mathrm{mJy} \mathrm{beam}^{-1}$ in $2012 \mathrm{Jan}$. and Feb., respectively. Given the very short observation time, we cannot make strong claims about the image fidelity and any significant variability. In both cases, the total cleaned flux density is consistent among the epochs with a value of $S_{8}=11.5 \mathrm{mJy}$, also similar to the result of our 2014 observations.

The astrometry of the 2000 and 2012 observations is not entirely consistent; there is a $\sim 10$ mas offset between the core peak at the two frequencies. The $8 \mathrm{GHz}$ observation positions are in good agreement (within $<1$ mas) with those reported at the same frequency by Doi et al. (2007).

\subsection{Model fits and proper motion}

We report in Table 1 the results of a model-fit in the image plane with elliptical Gaussian components to the 2000 and 2014 $5 \mathrm{GHz}$ images. We use four components to describe the 2000 data (which have better coverage of the $(u, v)$-plane) and three components for the 2014 data. In both cases, the main features at the two edges of the structure can be easily identified. Their positions are consistent to better than one beam and the flux densities are also consistent within the calibration uncertainty.

The separation between $E$ and $W$ changed from 9.4 mas in 2002 to 9.2 mas in 2014; since the difference is less than $1 / 5$ of the beam, we claim that both components are stationary, and that

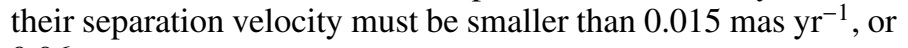
$0.06 c$.

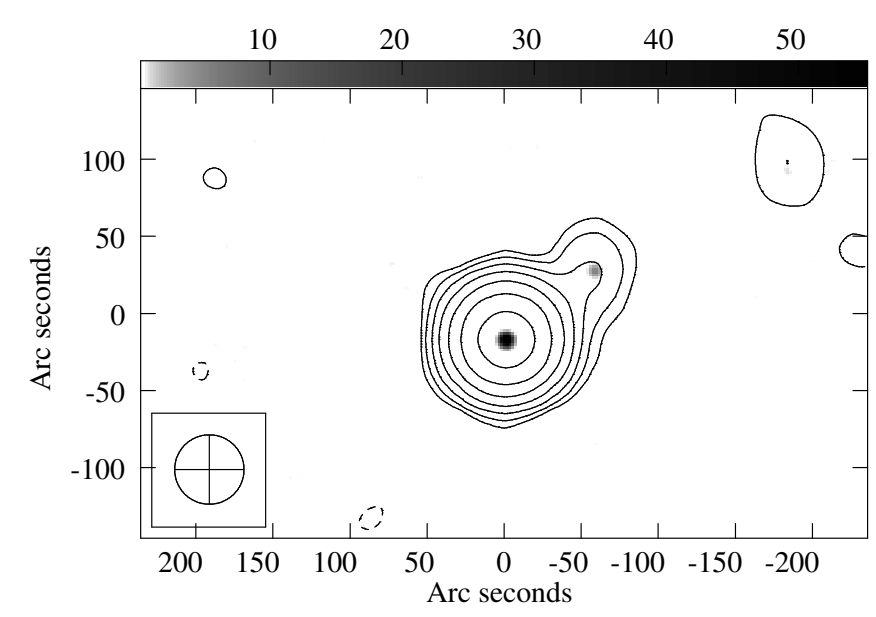

Fig. 5. Overlay of the NVSS (contours) and FIRST (grey scale) $1.4 \mathrm{GHz}$ VLA images. The NVSS HPBW is $45^{\prime \prime}$ and is shown in the bottom left corner; the FIRST HPBW is $4.5^{\prime \prime}$. Contours are traced at $(-1,1,2,4, \ldots) \times 1.3 \mathrm{mJy}^{-1}$ beam $^{-1}$; the grey scale is shown in the top wedge and spans the range between 0.4 and $55 \mathrm{mJy}^{-1}$ beam $^{-1}$.

Table 1. Results of the model-fit to the $5 \mathrm{GHz}$ images of IRAS $17020+4544$.

\begin{tabular}{llccc}
\hline \hline $\begin{array}{l}\text { Epoch } \\
\text { (year) }\end{array}$ & Component & $\begin{array}{c}x \\
\text { (mas) }\end{array}$ & $\begin{array}{c}y \\
\text { (mas) }\end{array}$ & $\begin{array}{c}S_{5} \\
\text { (mJy) }\end{array}$ \\
\hline 2000 & E & 0.0 & 0.0 & 17.9 \\
& A1 & -1.3 & -0.13 & 3.5 \\
& A2 & -3.9 & -0.6 & 2.4 \\
& W & -9.4 & -0.9 & 2.0 \\
2014 & E & 0.08 & -0.05 & 14.1 \\
& B & -3.1 & -1.9 & 5.2 \\
& W & -9.1 & -0.8 & 1.5 \\
\hline
\end{tabular}

Notes. The coordinates are referenced to the peak position of the brightest component in the 2000 combined images.

\section{Radio properties of IRAS $17020+4544$}

IRAS $17020+4544$ is also known as B3 $1702+457$, since it was detected in the B3 survey at $408 \mathrm{MHz}$ with the Bologna Northern Cross (Ficarra et al. 1985). As it is a B3 source, it is quite bright and indeed it is detected in many other surveys: the 6th Cambridge survey of radio sources (6C, Hales et al. 1988), the Texas survey of radio sources (Douglas et al. 1996), and the Westerbork Northern Sky Survey (WENSS, Rengelink et al. 1997) at low frequency; the NRAO VLA Sky Survey (NVSS, Condon et al. 1998) and the Faint Images of the Radio Sky at Twenty Centimeters (FIRST, Becker et al. 1995) at $1.4 \mathrm{GHz}$; the 87GB and GB6 at $5 \mathrm{GHz}$ (Gregory \& Condon 1991; Gregory et al. 1996). From its flux density and luminosity distance, the radio power of IRAS $17020+4544$ is $P_{150 \mathrm{MHz}}=$ $3.2 \times 10^{24} \mathrm{~W} \mathrm{~Hz}^{-1}\left(v L_{v}=4.8 \times 10^{39} \mathrm{erg} \mathrm{s}^{-1}\right)$ and $P_{1.4 \mathrm{GHz}}=$ $1.0 \times 10^{24} \mathrm{~W} \mathrm{~Hz}^{-1}\left(v L_{v}=1.5 \times 10^{40} \mathrm{erg} \mathrm{s}^{-1}\right)$, calculated at $150 \mathrm{MHz}$ from the $6 \mathrm{C}$ and at $1.4 \mathrm{GHz}$ from the NVSS values, respectively.

The FIRST image (grey tones in Fig. 5) reveals a bright, compact main component, unresolved at the $\sim 5^{\prime \prime}$ angular scale, and a fainter secondary component located at $1.2^{\prime}$ (81 kpc projected at the IRAS $17020+4544$ redshift), in PA $-52^{\circ}$, which is also visible in the NVSS image (contours in Fig. 5) as an extension of the main component. The flux density in the NVSS 
is only slightly higher than in the FIRST (121 vs. $118 \mathrm{mJy})$, indicating that the source is compact at this angular resolution; the total flux density in the NVSS might also be slightly affected by the secondary component, which is only partly resolved at the $45^{\prime \prime}$ angular resolution of the survey. The two components are both well separated in the FIRST, which can be used to determine their brightness ratio (118:6, or $\sim 20)$. We are thus confident that any flux density measured at lower resolution is only slightly affected by the presence of this component. A visual inspection of the SDSS images does not reveal any obvious optical counterpart, so it remains uncertain whether this is a background object or a large-scale feature associated with IRAS $17020+4544$ itself. We note that kiloparsec scale emission is not uncommon in NLS1s, having been discovered in ten objects to date (Antón et al. 2008; Doi et al. 2012, 2015; Gliozzi et al. 2010; Richards \& Lister 2015; Whalen et al. 2006). However, with $\sim 80 \mathrm{kpc}$ (projected) linear size, IRAS $17020+4544$ would be the largest NLS1 known so far. If the third weaker component located further north-west were also physically associated with IRAS $17020+4544$, the size would become even larger. Deep, low frequency observations with a few arcsecond angular resolution will be necessary to clarify this issue.

In addition to these $1.4 \mathrm{GHz}$ data, Snellen et al. (2004) considered a number of additional multifrequency interferometric and single dish datasets between $150 \mathrm{MHz}$ and $22 \mathrm{GHz}$. They report a spectral index of $\alpha=-0.88$, slightly flattening at low frequency, and provide constraints on the turnover frequency and peak flux density of $v_{\text {peak }}<150 \mathrm{MHz}$ and $S_{\text {peak }}>380 \mathrm{mJy}$, respectively. A low frequency turnover is confirmed by the flatter ( $\alpha=-0.23$ ) spectral index between 325 and $1400 \mathrm{MHz}$ reported by Massaro et al. (2014) and the non-detection at $74 \mathrm{MHz}$ in the VLA Low-Frequency Survey (VLSS, whose typical pointsource detection limit is $0.7 \mathrm{Jy}_{\text {beam }^{-1}}$, Cohen et al. 2007). On the basis of its spectrum and linear size, Snellen et al. (2004) classify the source as a candidate low power compact steep spectrum (CSS) source and include it in the Compact Radio sources at Low Redshift (CORALZ) sample ${ }^{1}$.

To construct their spectra, Snellen et al. (2004) considered data taken at the same frequency in different epochs, thus also providing us with variability information: only small deviations are present, suggesting that variability in the radio is not significant; if we take into account possible calibration uncertainties and different angular resolution in the various observations, the amount of real source variability has to be very limited, if any. In this sense, the most remarkable finding is the comparison of the single dish (87GB and GB6) and interferometric (VLA) $5 \mathrm{GHz}$ flux density values, with $S_{87 \mathrm{~GB}}=26 \pm 6 \mathrm{mJy}, S_{\mathrm{GB} 6}=29 \pm$ $4 \mathrm{mJy}$, and $S_{\mathrm{VLA}}=40 \pm 4 \mathrm{mJy}$. It is not clear how reliable the single dish measurements are since they are near the flux density limit of the catalogues ( 25 and $18 \mathrm{mJy}$ for the $87 \mathrm{~GB}$ and the GB6 data, respectively). As the $87 \mathrm{~GB}$ data were reported by many authors to estimate the radio loudness $(R)$, if the real flux density is larger than $26 \mathrm{mJy}$, then the real $R$ could also be accordingly higher by $\Delta \log R \sim+0.2$. On the issue of radio loudness, it is interesting to note that the values of $R$ reported in the literature vary significantly in different works, typically based on the assumptions on the optical spectrum used to estimate the $B$ magnitude. The most recent value is $\log R=2.17$ (Doi et al. 2016), based on the ratio of FIRST $1.4 \mathrm{GHz}$ radio to

\footnotetext{
1 However, later high angular resolution observations of the full sample (de Vries et al. 2009) do not include the source; no reason for this choice is given in the paper.
}

SDSS $g$-band. Such values put IRAS $17020+4544$ firmly in the radio loud regime.

At high angular resolution, we can compare our new and reprocessed VLBA data with other VLBI observations, in particular the $8.4 \mathrm{GHz}$ Japanese VLBI Network (JVN) observations by Doi et al. (2007) and the 1.6 VLBA data by Doi et al. (2011). These two datasets show a fairly resolved morphology, which accounts for over one-half of the total flux density measured at the same frequency, for example by the VLA. In particular, $S_{1.6}=79.9 \pm 4.0 \mathrm{mJy}$ and $S_{8.4}=18.5 \pm 2.6 \mathrm{mJy}$. The resulting non-simultaneous spectral index is $\alpha=0.84 \pm 0.21$, consistent with the value found with the simultaneous data. A further nondetection at $22 \mathrm{GHz}$ at the $<9 \mathrm{mJy}$ level, entirely consistent with our result, is also reported by Doi et al. (2016).

\section{Discussion}

Based on the information gathered by the new, the archival, and the literature data on IRAS $17020+4544$, we determine that it is a jetted non-thermal source. The steep overall spectrum on $\sim \operatorname{kpc}(\alpha=0.9$, Snellen et al. 2004) and $\sim$ pc ( $\alpha=1.0$, this work) scales indicates that the radio emission has a synchrotron origin. The total radio luminosity $\left(P_{1.4 \mathrm{GHz}}=1.0 \times 10^{24} \mathrm{~W} \mathrm{~Hz}^{-1}\right)$ and, above all, the brightness temperature of the VLBI main component $\left(T_{b}=1.0 \times 10^{8} \mathrm{~K}\right)$ are far too large for starburst processes, and constrain the radio emission to have predominantly a nuclear origin. The difference between the VLBI and total flux density reveals the presence of radio emission on scales ranging from $\mathrm{pc}$ to kpc most likely fuelled by the central region. Taken together, these elements indicate that the black hole in IRAS 17020+4544 is powering a non-thermal plasma jet in addition to the X-ray outflow of gas studied by Longinotti et al. (2015).

From this starting point, we can then can try to classify the source in a few different ways that are complementary rather than conflicting. As already proposed by Snellen et al. (2004), the compact structure and steep integrated radio spectrum suggest that IRAS $17020+4544$ is a candidate CSS source. Unlike compact flat-spectrum sources, such as blazars, CSS sources are oriented on the plane of the sky, so their compactness is intrinsic rather than due to projection effects, as highlighted by the lack of a prominent compact flat-spectrum core. CSS sources are thought to be the early stages of evolution of the more extended, classical FR2 radio galaxies, as indicated by their young kinematic and spectral age estimates (Fanti et al. 1995; Murgia et al. 1999; Giroletti \& Polatidis 2009). The parsec scale structure presented in this work is in agreement with the lack of a prominent core and of strong beaming effects; however, the absence of component motion over a 14 yr time scale does not indicate that this source is in a state of rapid growth, which would be typical of CSS sources. Moreover, the radio luminosity of IRAS $17020+4544$ is also significantly lower $\left(\sim 10^{-4} \times\right)$ than that of the archetypical CSS sources, suggesting that it could lie in a different evolutionary path.

Giroletti et al. (2005) and Kunert-Bajraszewska et al. (2010) have indeed introduced the class of low-power compact (LPC) or low-luminosity compact (LLC) objects, which - like the CSS sources - are thought to be intrinsically compact, yet they present lower luminosities $\left(P_{1.4 \mathrm{GHz}}<10^{26} \mathrm{~W} \mathrm{~Hz}^{-1}\right)$ and slower or null advance velocities. These sources might be shortlived objects or the progenitors of low luminosity FR1 radio sources. In terms of radio properties (morphology, spectrum, power), IRAS $17020+4544$ fits well within this class; however, its peculiar optical properties do not match the typical features of a FR1 progenitor: the host is a barred spiral galaxy 
(Ohta et al. 2007) and the optical spectrum characterizes the source as a NLS1.

The presence of radio emission in NLS1 has attracted a lot of interest in recent times, especially after the discovery of gamma-ray emission from a growing number of RL NLS1s (Abdo et al. 2009a,b; D'Ammando et al. 2012, 2015). Gamma-ray emission in NLS1s is ascribed to the presence of a beamed relativistic jet, similar to what happens in blazars, although some similarities with CSS sources in spectrum and size have been claimed, e.g. in PKS 2004-447 (Gallo et al. 2006; Orienti et al. 2015; Schulz et al. 2016) and SDSS J143244.91+301435.3 (Caccianiga et al. 2014). Evidence of beaming in RL NLS1s in general (not only in gamma-ray NLS1s) has been found in several objects, in the form of dominant cores, high brightness temperatures (above $10^{10} \mathrm{~K}$, e.g. Giroletti et al. 2011; Wajima et al. 2014; Gu et al. 2015), and occasionally superluminal motion (D'Ammando et al. 2012). Our results on the morphology and brightness temperature indicate that IRAS $17020+4544$ is different from this subset of the NLS1 population; yet, it also remains markedly separated from the bulk of the radio quiet NLS1 population, which have much lower radio powers (as low as $P_{1.4 \mathrm{GHz}} \sim 10^{19} \mathrm{~W} \mathrm{~Hz}^{-1}$ ) and are seldom detected with VLBI (Giroletti \& Panessa 2009; Doi et al. 2013). One intriguing possibility is that IRAS $17020+4544$ belongs to the elusive population of misaligned RL NLS1s (Berton et al. 2015).

From a multiwavelength perspective, IRAS $17020+4544$ is also highly remarkable for its X-ray properties. While its X-ray and bolometric luminosities are not exceptional $\left(L_{0.3-10 \mathrm{keV}} \sim\right.$ $\left.1.5 \times 10^{44} \mathrm{erg} \mathrm{s}^{-1}, L_{\mathrm{BOL}} \sim 5.2 \times 10^{44} \mathrm{erg} \mathrm{s}^{-1}\right)$, the recent high spectral resolution $(E / \Delta E \sim 1000) X M M-N e w t o n$ observations of Longinotti et al. (2015), obtained within one month of our VLBA data, have revealed a unique subrelativistic outflow. The spectrum shows a series of absorption lines corresponding to at least five absorption components with velocities in the range of 23000-33000 $\mathrm{km} \mathrm{s}^{-1}$; the associated column densities observed in the X-ray wind span from $5 \times 10^{20}$ to $\sim 10^{24} \mathrm{~cm}^{-2}$. The mass and energy output estimated by these authors indicate that the higher column density wind is capable of expelling sufficient gas to produce feedback onto the host galaxy, even if the source is $\sim 100$ times less luminous than quasars where this phenomenon is commonly observed (Nardini et al. 2015; Tombesi et al. 2015). Whether the presence of radio emission is somehow related to this peculiar wind is a question worth exploring. In a similar case of coexistence of fast X-ray outflow and radio jet, albeit in a high radio power source, Tombesi et al. (2012) reported that the pressure estimated by the X-ray column density is comparable to the pressure of the radio inner jet, therefore lending support to the idea that the X-ray outflow may help to collimate the jet. In the case of IRAS $17020+4544$, higher resolution VLBA observations will provide a better estimate of the spatial scale of the jetted structure and its properties, shedding light on the possible wind-jet connection.

The mutual interaction between AGN winds and jets is the subject of much debate. Zakamska \& Greene (2014) found a correlation between radio power and gas kinematics (in particular, [OIII] line velocity width) in a sample of luminous type-2 radio quiet quasars. They considered several scenarios to interpret this correlation, and concluded that either radiatively driven winds propagate into the interstellar medium of the host galaxy, generating shock fronts which in turn accelerate particles producing radio emission, or mechanical energy of relativistic jets heats overpressured cocoons, which then launch winds of ionized gas (Mullaney et al. 2013). The detection of the X-ray outflow in
IRAS $17020+4544$ and the source proximity would in principle permit us to study this issue with adequate linear resolution. However, the radio luminosity $\log v L_{v}=40.2$ ([erg s$\left.{ }^{-1}\right]$, calculated at $v=1.4 \mathrm{GHz}$ ) is higher than the bolometric luminosity, confirming that IRAS $17020+4544$ is a radio-loud source and therefore quite exceptional with respect to the radio-quiet population studied by Zakamska \& Greene (2014).

\section{Summary}

The results of our study can be summarized as follows:

- The radio luminosity $\left(\log L_{150}=24.5 \mathrm{~W} \mathrm{~Hz}^{-1}\right)$ is typical of a FR1 radio galaxy, but the structure is compact, i.e. $<\mathrm{kpc}$ scale. As already hinted at by other works (Snellen et al. 2004), it meets the criteria for a low power compact source (LPC, Giroletti et al. 2005).

- The integrated steep spectrum $(\alpha=-0.9)$ clearly indicates that the radio emission has a synchrotron origin, revealing the presence of relativistic particles and magnetic fields.

- On parsec scales, the images at 5 and $8 \mathrm{GHz}$ indicate that the source is clearly extended in an amorphous yet predominantly linear structure, indicating the presence of a jet or outflow on scales of about 10 parsecs; the non-detections at 15 and $24 \mathrm{GHz}$, when compared with the VLA flux densities at the same frequency, also indicate that the source structure is heavily resolved.

- Within this diffuse emission, there is a main component that is brighter, which would naturally be identified with the "core"; however, it is not characterized by a classical flat/inverted spectrum.

- The lack of a prominent flat spectrum component suggests that the amount of beaming in any possible core is negligible, i.e. the plasma does not have a bulk relativistic motion or it is moving at a very large angle to the line of sight, which is also in agreement with the lack of variability.

- The lack of motion over $15 \mathrm{yr}$ suggests that the separation speed is lower than $0.2 \mathrm{mas} / 14 \mathrm{yr}$, i.e. $<0.06 c$.

All these properties classify IRAS $17020+4544$ as a unique object and not easily classifiable as a standard radio emitting AGN. In addition IRAS $17020+4544$ is an optimal laboratory for the detailed study of AGN feedback. The coexistence of an ultrafast X-ray wind and a molecular large-scale outflow indicate that this process is in action via an induced shock. The peculiar radio properties are likely related to this phenomenon. Future radio observations up to $24 \mathrm{GHz}$ in phase reference mode will allow us to obtain higher fidelity total intensity and spectral images, useful to investigate the role of the radio emission in this context.

Acknowledgements. We acknowledge financial contribution from grant PRININAF-2014. This research has made use of NASA's Astrophysics Data System Bibliographic Services. This research has made use of the NASA/IPAC Extragalactic Database (NED), which is operated by the Jet Propulsion Laboratory, California Institute of Technology, under contract with the National Aeronautics and Space Administration. The National Radio Astronomy Observatory is a facility of the National Science Foundation operated under cooperative agreement by Associated Universities, Inc.

\section{References}

Abdo, A. A., Ackermann, M., Ajello, M., et al. 2009a, ApJ, 699, 976 Abdo, A. A., Ackermann, M., Ajello, M., et al. 2009b, ApJ, 707, L142 Antón, S., Browne, I. W. A., \& Marchã, M. J. 2008, A\&A, 490, 583 Becker, R. H., White, R. L., \& Helfand, D. J. 1995, ApJ, 450, 559 Berton, M., Foschini, L., Ciroi, S., et al. 2015, A\&A, 578, A28 
Caccianiga, A., Antón, S., Ballo, L., et al. 2014, MNRAS, 441, 172

Chartas, G., Brandt, W. N., Gallagher, S. C., \& Garmire, G. P. 2002, ApJ, 579, 169

Cohen, A. S., Lane, W. M., Cotton, W. D., et al. 2007, AJ, 134, 1245

Condon, J. J., Cotton, W. D., Greisen, E. W., et al. 1998, AJ, 115, 1693

D’Ammando, F., Orienti, M., Finke, J., et al. 2012, MNRAS, 426, 317

D’Ammando, F., Orienti, M., Larsson, J., \& Giroletti, M. 2015, MNRAS, 452, 520

de Vries, N., Snellen, I. A. G., Schilizzi, R. T., Mack, K.-H., \& Kaiser, C. R. 2009, A\&A, 498, 641

Di Matteo, T., Springel, V., \& Hernquist, L. 2005, Nature, 433, 604

Doi, A., Fujisawa, K., Inoue, M., et al. 2007, PASJ, 59, 703

Doi, A., Asada, K., \& Nagai, H. 2011, ApJ, 738, 126

Doi, A., Nagira, H., Kawakatu, N., et al. 2012, ApJ, 760, 41

Doi, A., Asada, K., Fujisawa, K., et al. 2013, ApJ, 765, 69

Doi, A., Wajima, K., Hagiwara, Y., \& Inoue, M. 2015, ApJ, 798, L30

Doi, A., Oyama, T., Kono, Y., et al. 2016, PASJ, 68, 73

Douglas, J. N., Bash, F. N., Bozyan, F. A., Torrence, G. W., \& Wolfe, C. 1996, AJ, 111, 1945

Fanti, C., Fanti, R., Dallacasa, D., et al. 1995, A\&A, 302, 317

Faucher-Giguère, C.-A., \& Quataert, E. 2012, MNRAS, 425, 605

Ficarra, A., Grueff, G., \& Tomassetti, G. 1985, A\&AS, 59, 255

Fukumura, K., Tombesi, F., Kazanas, D., et al. 2014, ApJ, 780, 120

Gallo, L. C., Edwards, P. G., Ferrero, E., et al. 2006, MNRAS, 370, 245

Giroletti, M., \& Panessa, F. 2009, ApJ, 706, L260

Giroletti, M., \& Polatidis, A. 2009, Astron. Nachr., 330, 193

Giroletti, M., Giovannini, G., \& Taylor, G. B. 2005, A\&A, 441, 89

Giroletti, M., Paragi, Z., Bignall, H., et al. 2011, A\&A, 528, L11

Gliozzi, M., Papadakis, I. E., Grupe, D., et al. 2010, ApJ, 717, 1243

Gregory, P. C., \& Condon, J. J. 1991, ApJS, 75, 1011
Gregory, P. C., Scott, W. K., Douglas, K., \& Condon, J. J. 1996, ApJS, 103, 427 Gu, M., \& Chen, Y. 2010, AJ, 139, 2612

Gu, M., Chen, Y., Komossa, S., et al. 2015, ApJS, 221, 3

Hales, S. E. G., Baldwin, J. E., \& Warner, P. J. 1988, MNRAS, 234, 919

Longinotti, A. L., Krongold, Y., Guainazzi, M., et al. 2015, ApJ, 813, L39

Komatsu, E., Dunkley, J., Nolta, M. R., et al. 2009, ApJS, 180, 330

Kunert-Bajraszewska, M., Gawroński, M. P., Labiano, A., \& Siemiginowska, A. 2010, MNRAS, 408, 2261

Massaro, F., Giroletti, M., D’Abrusco, R., et al. 2014, ApJS, 213, 3

Mullaney, J. R., Alexander, D. M., Fine, S., et al. 2013, MNRAS, 433, 622

Murgia, M., Fanti, C., Fanti, R., et al. 1999, A\&A, 345, 769

Nardini, E., Reeves, J. N., Gofford, J., et al. 2015, Science, 347, 860

Ohta, K., Aoki, K., Kawaguchi, T., \& Kiuchi, G. 2007, ApJS, 169, 1

Orienti, M., D’Ammando, F., Larsson, J., et al. 2015, MNRAS, 453, 4037

Pounds, K. A., Reeves, J. N., King, A. R., et al. 2003, MNRAS, 345, 705

Rengelink, R. B., Tang, Y., de Bruyn, A. G., et al. 1997, A\&AS, 124, 259

Richards, J. L., \& Lister, M. L. 2015, ApJ, 800, L8

Schulz, R., Kreikenbohm, A., Kadler, M., et al. 2016, A\&A, 588, A146

Snellen, I. A. G., Mack, K.-H., Schilizzi, R. T., \& Tschager, W. 2004, MNRAS, 348,227

Tchekhovskoy, A., Narayan, R., \& McKinney, J. C. 2011, MNRAS, 418, L79

Tombesi, F., Sambruna, R. M., Marscher, A. P., et al. 2012, MNRAS, 424, 754

Tombesi, F., Tazaki, F., Mushotzky, R. F., et al. 2014, MNRAS, 443, 2154

Tombesi, F., Meléndez, M., Veilleux, S., et al. 2015, Nature, 519, 436

Wajima, K., Fujisawa, K., Hayashida, M., et al. 2014, ApJ, 781, 75

Wang, T., \& Lu, Y. 2001, A\&A, 377, 52

Whalen, D. J., Laurent-Muehleisen, S. A., Moran, E. C., \& Becker, R. H. 2006, AJ, 131, 1948

Zakamska, N. L., \& Greene, J. E. 2014, MNRAS, 442, 784

Zubovas, K., \& King, A. 2012, ApJ, 745, L34 